\title{
Space-Time Coded Systems using Continuous Phase Modulation
}

\author{
Rachel L. Maw, Student Member, IEEE, and Desmond P. Taylor, Life Fellow, IEEE
}

\begin{abstract}
We develop space-time trellis coded (STC) schemes using continuous-phase modulation (CPM). We employ the Rimoldi model of CPM to create a decomposed model of STC-CPM. The decomposition separates the coding from the modulation. The space-time encoding and the inherent CPM encoding is combined into a single trellis encoder on the ring of integers modulo- $p$. This is followed by a bank of memoryless modulators. The model allows the search for good space-time codes to take into account the inherent encoding of the modulation.
\end{abstract}

Index Terms-Continuous phase modulation, space-time coding, ring convolutional codes.

\section{INTRODUCTION}

$\mathbf{S}$ PACE-TIME coding (STC) has been widely used to reduce the effects of multipath fading [1], [2]. Most STC systems are based on linear modulations; however, it was shown in [3]-[6] that continuous phase modulation [7] (CPM) is a good alternative. Its constant envelope allows the use of lowcost power-efficient nonlinear amplifiers. The phase continuity implies that the modulation has memory or inherent coding and, therefore, a trellis representation.

Space-time code design with CPM has been investigated in [8] and [9], where design rules and code constructions for specific forms of CPM are derived that guarantee full spatial diversity. Trellis code design rules are presented in [10] for general constant envelope space-time modulation. Interleaved externally encoded STC-CPM systems have been developed in [11]-[13]. A reduced complexity receiver for layered spacetime schemes with minimum-shift keying (MSK)-like modulations is presented in [14].

In this paper, an STC-CPM system that exploits the Rimoldi model [15] of CPM is developed for $M$-ary modulation with $M=p^{k_{m}}$, where $p$ and $k_{m}$ are positive integers. We assume a rational modulation index $h=q / p$, and form a decomposed model of STC-CPM. The resulting system consists of a convolutional encoder on the integer ring $\mathbb{Z}_{p}$ [16], [17], followed by a bank of $L_{t}$ memoryless modulators, where $L_{t}$ is the number of transmit antennas. This can be seen as an extension of the work in [18] and [19] to multiple transmit antenna schemes. Decoding is accomplished using the Viterbi algorithm on the trellis of the encoder. The STC-CPM encoder could be

Paper approved by H. Jafarkhani, the Editor for Modulation/Detection of the IEEE Communications Society. Manuscript received July 17, 2006; revised November 21, 2006. This work was supported in part by the Bright Futures Scholarship Scheme administered by the New Zealand Tertiary Education Commission and in part by the Foundation for Research, Science and Technology. This paper was presented in part at the WIRELESSCOM, Maui, HI, June 2005 and at GLOBECOM, St. Louis, MO, November 2005.

The authors are with the Department of Electrical and Computer Engineering, University of Canterbury, Christchurch 8140, New Zealand (e-mail: rlm57@student.canterbury.ac.nz; taylor@elec.canterbury.ac.nz).

Digital Object Identifier 10.1109/TCOMM.2007.908508 used as the inner code of an interleaved iteratively decoded structure [11]-[13]. In [20], serially concatenated CPM structures that utilize ring convolutional codes as the outer codes and CPM as the inner code are investigated for single thread systems.

The decomposition allows us to investigate code design for STC-CPM on an overall encoder trellis, which incorporates both the coding inherent to the modulation and that of the STC. For linearly modulated space-time trellis coded schemes, the code design criteria depend on $\rho L_{r}$ [21], [22], where $L_{r}$ is the number of receive antennas and $\rho$ is the minimum rank of the code word distance matrices with $\rho \leq L_{t}$. If $\rho L_{r} \leq 3$, the rank and the minimum determinant [1] of the distance matrices are the appropriate criteria. If $\rho L_{r}>3$, the minimum trace of the code word distance matrices or, equivalently, the minimum squared Euclidean distance $\left(d_{\mathrm{min}}^{2}\right)$ between the transmitted signals is better used [21], [22]. Directly analogous results apply to STC-CPM, if the signal distance matrix [9] is considered. ${ }^{1}$ The rank, determinant and $d_{\mathrm{min}}^{2}$ criteria then hold for STC-CPM. Using the decomposed model, we may derive an expression for $d_{\mathrm{min}}^{2}$ based on the trellis of the overall encoder [5].

We give a brief overview of CPM in the following section. We then develop the STC-CPM system model. Simulated performance curves are presented and discussed in Section IV.

\section{Continuous Phase Modulation}

A CPM signal [7], [15] can be described by

$$
\begin{array}{r}
s(t, \boldsymbol{X}(D))=\sqrt{\frac{2 E}{T}} \cos \left(2 \pi f_{1} t+\tilde{\psi}(t, \boldsymbol{X}(D))+\psi_{o}\right) \\
n T \leq t \leq(n+1) T
\end{array}
$$

where $f_{1}=f_{c}-(M-1) h / 2 T$ is the asymmetric carrier frequency, $f_{c}$ is the carrier frequency, $E$ is the symbol energy, $T$ is the symbol period, $\psi_{o}$ is the initial phase offset, ${ }^{2}$ and the $M$-ary data sequence $\boldsymbol{U}$ has elements $U_{n} \in\{0,1, \ldots,(M-1)\}$. The information carrying or physical tilted phase is given by

$$
\begin{aligned}
\tilde{\psi}(t, \boldsymbol{X}(D)) & =\left(2 \pi h\left[\sum_{i=0}^{n-L} U_{i}\right] \bmod p+4 \pi h \sum_{i=n}^{n-L+1} U_{i} q(t-i T)\right. \\
+ & W(t)) \bmod (2 \pi), \quad n T \leq t \leq(n+1) T
\end{aligned}
$$

\footnotetext{
${ }^{1}$ We note that in any practical sense, CPM signals and codes are indistinguishable since the phase variation within each interval defines the code and, hence, the distance matrix.

${ }^{2}$ This is set arbitrarily to zero in what follows.
} 
where $h=q / p$ is the modulation index, $W(t)$ is the sum of data independent terms, and $q(t)$ is the phase function. Function $q(t)$ is the integral of the instantaneous frequency response and has a duration of $L T$ [15]. From (2), we see that the output of the modulator is specified by the vector

$$
\boldsymbol{X}_{n}=\left[U_{n}, \ldots, U_{n-L+1}, V_{n}\right], \quad n T \leq t \leq(n+1) T
$$

where

$$
\begin{array}{r}
V_{n}=\left[\sum_{i=0}^{n-L} U_{i}\right] \bmod p=\left[V_{n-1}+U_{n-L}\right] \bmod p, \\
n T \leq t \leq(n+1) T .
\end{array}
$$

CPM can thus be represented by a $p M^{L-1}$ state time-invariant phase trellis.

We may represent the $M$-ary $\left(M=p^{k_{m}}\right)$ input symbols in radix- $p$ form as

$$
U_{n}=\sum_{j=1}^{k_{m}} U_{n}^{j} p^{k_{m}-j}, \quad n T \leq t \leq(n+1) T
$$

where the subsymbols $U_{n}^{j}$ are on $\mathbb{Z}_{p}$. We denote the $j$ th subsymbol sequence as $\boldsymbol{U}^{j}(D)=U_{0}^{j}+U_{1}^{j} D+\cdots+U_{n}^{j} D^{n}+\cdots$, where $D$ represents a unit delay. A convolutional encoder on $\mathbb{Z}_{p}$ can then generate $\boldsymbol{X}(D)=\boldsymbol{X}_{0}+\boldsymbol{X}_{1} D+\cdots+\boldsymbol{X}_{n} D^{n}+\cdots$, to form the input to a time-invariant memoryless modulator that outputs $s(t, \boldsymbol{X}(D))$. This encoder encodes the sequences $\boldsymbol{U}(D)=\left[\begin{array}{ll}\boldsymbol{U}^{1}(D) & \boldsymbol{U}^{2}(D) \ldots \boldsymbol{U}^{k_{m}}(D)\end{array}\right]$, and has the $k_{m} \times\left(k_{m} L+1\right)$ generator matrix given by (3), where $\mathbf{0}_{i, j}$ represents an $(i \times j)$ matrix of zeroes. The output of the encoder is related to the input by $\boldsymbol{X}(D)=\boldsymbol{C}(D) \boldsymbol{U}(D)$.

To avoid catastrophic codes in what follows, we use a precoder as in [18] and [23] to cancel the feedback term in $\boldsymbol{C}(D)$. The precoder generator matrix is given by

$$
\boldsymbol{T}(D)=\left[\begin{array}{cc}
\boldsymbol{I}_{k_{m}-1} & \mathbf{0}_{k_{m}-1,1} \\
\mathbf{0}_{1, k_{m}-1} & 1-D
\end{array}\right]
$$

where $\boldsymbol{I}_{i}$ is the $(i \times i)$ identity matrix. $\boldsymbol{T}(D)$ is cascaded with $\boldsymbol{C}(D)$ to create a feedback-free CPE (FF-CPE) with the generator matrix $\boldsymbol{W}(D)=\boldsymbol{T}(D) \boldsymbol{C}(D)$.

\section{STC-CPM}

The proposed space-time coded CPM transmitter structure is shown in Fig. 1. We let $L_{t}$ and $L_{r}$ denote the number of transmit antennas and receive antennas, respectively. We define the space-time encoder as a linear rate- $k / l$ convolutional encoder on the integer ring $\mathbb{Z}_{p}$, where $k, l$ are integers and $l \geq k$. The

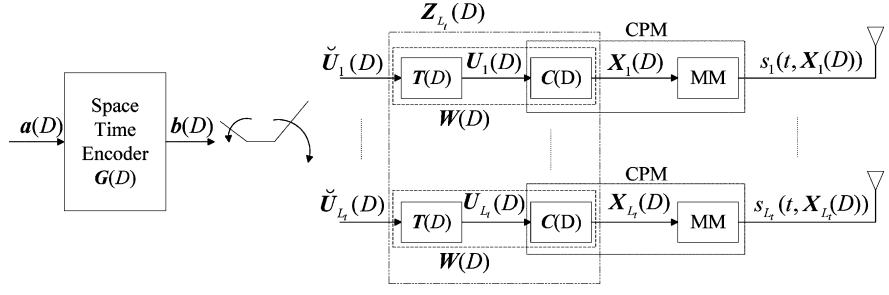

Fig. 1. Block diagram of the STC-CPM transmitter.

space-time encoder is followed by a commutator that produces the input sequences for the $L_{t} \mathrm{FF}$-CPEs. The $p$-ary data sequence $\boldsymbol{a}(D)=\boldsymbol{a}_{0}+\boldsymbol{a}_{1} D+\cdots+\boldsymbol{a}_{j} D^{j}+\cdots$ is input into the spacetime encoder, which has generator matrix $\boldsymbol{G}(D)$. During the in-

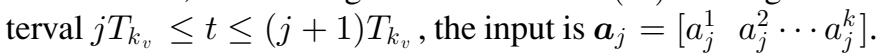
The space-time encoder output is then

$$
\boldsymbol{b}(D)=\boldsymbol{a}(D) \boldsymbol{G}(D)
$$

where $\boldsymbol{b}(D)=\boldsymbol{b}_{0}+\boldsymbol{b}_{1} D+\cdots+\boldsymbol{b}_{j} D^{j}+\cdots$ and $\boldsymbol{b}_{j}=\left[b_{j}^{1} b_{j}^{2}\right.$ $\left.\cdots b_{j}^{l}\right]$ is output during the interval $j T_{k_{v}} \leq t \leq(j+1) T_{k_{v}}$.

The commutator groups the STC output symbols into $L_{t}$ blocks, each containing $k_{m} p$-ary symbols to form the input for the FF-CPEs. During the $n$th symbol interval of duration $T=\left(T_{k_{v}} k_{m} L_{t}\right) / l$, the resulting $k_{m} L_{t}$ dimensional vector of $p$-ary symbols is given by

$$
\breve{\boldsymbol{U}}_{n}=\left[\breve{\boldsymbol{U}}_{1, n} \breve{\boldsymbol{U}}_{2, n} \cdots \breve{\boldsymbol{U}}_{L_{t}, n}\right], \quad n T \leq t \leq(n+1) T
$$

where each subvector

$$
\breve{\boldsymbol{U}}_{i, n}=\left[\breve{U}_{i, n}^{1} \breve{U}_{i, n}^{2} \cdots \breve{U}_{i, n}^{k_{m}}\right], \quad i=1,2, \ldots, L_{t}
$$

consists of the $k_{m}$ coefficients of the radix- $p$ expression of (6). A subvector forms one $M$-ary channel symbol. The output sequence of the $i$ th FF-CPE may then be written as

$$
\boldsymbol{U}_{i}(D)=\breve{\boldsymbol{U}}_{i}(D) \boldsymbol{W}(D), \quad i=1,2, \ldots, L_{t}
$$

where $\breve{\boldsymbol{U}}_{i}(D)=\breve{\boldsymbol{U}}_{i, 0}+\breve{\boldsymbol{U}}_{i, 1} D+\cdots+\breve{\boldsymbol{U}}_{i, j} D^{j}+\cdots \quad$ and $\boldsymbol{X}_{i}(D)=\boldsymbol{X}_{i, 0}+\boldsymbol{X}_{i, 1} D+\cdots+\boldsymbol{X}_{i, j} D^{j}+\cdots$, so that the $L k_{m}+1$-symbol vector output during the $n$th symbol interval is given by

$$
\begin{array}{r}
\boldsymbol{X}_{i, n}=\left[\begin{array}{llllll}
X_{i, n}^{1,1} \cdots X_{i, n}^{1, k_{m}} X_{i, n}^{2,1} & \cdots & X_{i, n}^{L, k_{m}} & X_{i, n}^{L+1,1}
\end{array}\right] \\
n T \leq t \leq(n+1) T .
\end{array}
$$

The signal $s_{i}\left(t, \boldsymbol{X}_{i}(D)\right)$ is transmitted from the antenna $i$. The throughput of the overall system is $\left(k \log _{2}(p) k_{m} L_{t}\right) / l$ bits per channel use.

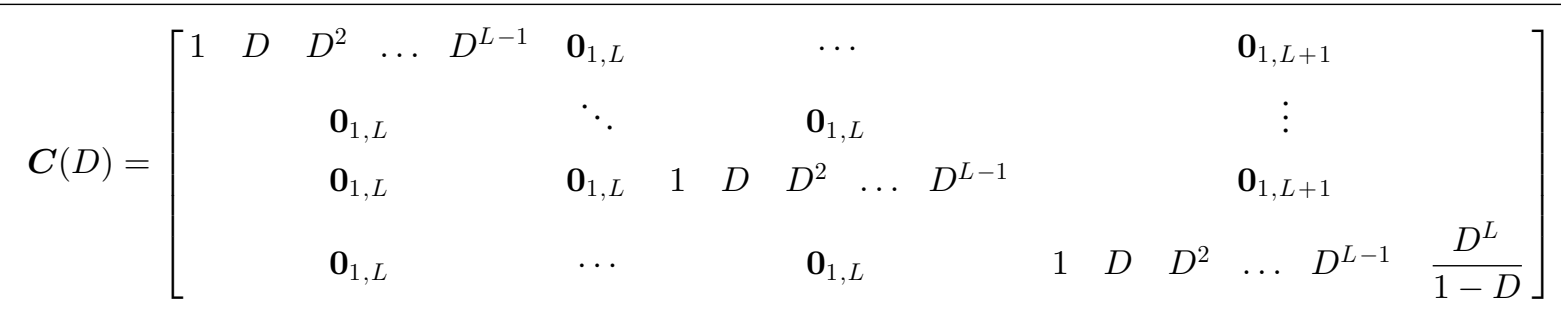




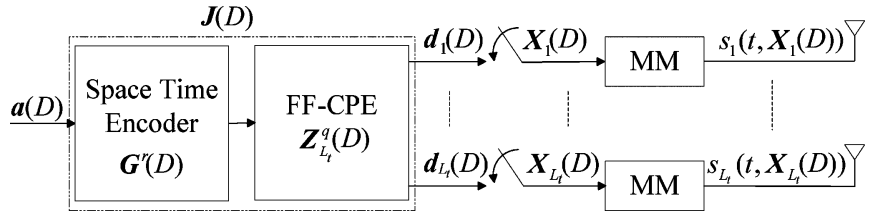

Fig. 2. Block diagram of the combined STC-CPM encoder.

We can combine the $L_{t}$ feedback-free CPEs $\boldsymbol{W}(D)$ into a single encoder with the $k_{m} L_{t} \times\left(k_{m} L+1\right) L_{t}$ generator matrix $Z_{L_{t}}(D)$

$$
=\left[\begin{array}{cccc}
\boldsymbol{W}(D) & \mathbf{0}_{k_{m}, k_{m} L+1} & \cdots & \mathbf{0}_{k_{m}, k_{m} L+1} \\
\mathbf{0}_{k_{m}, k_{m} L+1} & \ddots & \ddots & \vdots \\
\vdots & \ddots & \boldsymbol{W}(D) & \mathbf{0}_{k_{m}, k_{m} L+1} \\
\mathbf{0}_{k_{m}, k_{m} L+1} & \cdots & \mathbf{0}_{k_{m}, k_{m} L+1} & \boldsymbol{W}(D)
\end{array}\right] .
$$

During the $n$th symbol interval, the input to $Z_{L_{t}}(D)$ is the $k_{m} L_{t}$ dimensional vector $\breve{\boldsymbol{U}}_{n}$ and the output is the $\left(k_{m} L+1\right) L_{t}$ dimensional vector

$$
\boldsymbol{X}_{n}=\left[\begin{array}{llll}
\boldsymbol{X}_{1, n} & \boldsymbol{X}_{2, n} \cdots \boldsymbol{X}_{L_{t}, n}
\end{array}\right], \quad n T \leq t \leq(n+1) T .
$$

We may combine $\boldsymbol{Z}_{L_{t}}(D)$ with the space-time encoder $\boldsymbol{G}(D)$ to obtain an encoder that incorporates the entire encoding process. If the rate- $k / l$ space-time encoder is designed such that $l=k_{m} L_{t}, \boldsymbol{G}(D)$ can be directly cascaded with $\boldsymbol{Z}_{L_{t}}(D)$. The overall generator matrix is then

$$
\boldsymbol{J}(D)=\boldsymbol{G}(D) \boldsymbol{Z}_{L_{t}}(D) .
$$

If $l \neq k_{m} L_{t}$, we must effectively move the commutator to follow the encoding as shown in Fig. 2. To do this, we find the lowest common multiple of $l$ and $k_{m} L_{t}$, such that $r l=q k_{m} L_{t}$, where $r$ and $q$ are integers. We convert $\boldsymbol{G}(D)$ to the rate-rk/rl matrix $\boldsymbol{G}^{r}(D)$ and $\boldsymbol{Z}_{L_{t}}(D)$ to the rate- $q k_{m} L_{t} / q L_{t}\left(k_{m} L+1\right)$ matrix $\boldsymbol{Z}_{L_{t}}^{q}(D)$. These may be directly cascaded to obtain the composite encoder $\boldsymbol{J}(D)=\boldsymbol{G}^{r}(D) \boldsymbol{Z}_{L_{t}}^{q}(D)$ that has an $r k \times$ $q L_{t}\left(k_{m} L+1\right)$ generator matrix. Only one rate conversion is required if $l$ is a multiple of $k_{m} L_{t}$ or vice versa. The encoder $\boldsymbol{G}^{r}(D)$ accepts the $r k$-symbol input vector

$$
\boldsymbol{a}_{j}=\left[\begin{array}{llll}
\mathrm{a}_{j}^{1} & a_{j}^{2} & \cdots & a_{j}^{r k}
\end{array}\right], \quad j T_{k_{f}} \leq t \leq(j+1) T_{k_{f}}
$$

and outputs an $r l$-symbol vector denoted $\boldsymbol{c}_{j}$ every $T_{k_{f}}$-second interval, where $T_{k_{f}}=r T_{k_{v}}=T q$. Similarly, the encoder $\boldsymbol{Z}_{L_{t}}^{q}(D)$ accepts the $q k_{m} L_{t}=r l$-symbol vector $\boldsymbol{c}_{j}$, output from $\boldsymbol{G}^{r}(D)$, and produces a $q L_{t}\left(k_{m} L+1\right)$-symbol vector given by

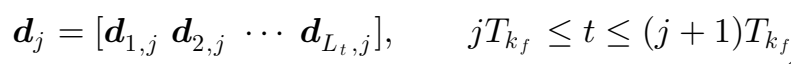

every $T_{k_{f}}$-second interval, where

$$
\begin{aligned}
\boldsymbol{d}_{i, j}= & {\left[\begin{array}{llll}
d_{i, j}^{1} & d_{i, j}^{2} & \cdots & d_{i, j}^{q\left(k_{m} L+1\right)}
\end{array}\right] } \\
= & {\left[\boldsymbol{X}_{i, j q} \boldsymbol{X}_{i, j q+1} \cdots \boldsymbol{X}_{i,(j+1) q-1}\right], } \\
& \quad i=1,2, \ldots, L_{t}, \quad j T_{k_{f}} \leq t \leq(j+1) T_{k_{f}}
\end{aligned}
$$

and

$$
\begin{aligned}
\boldsymbol{X}_{i, n}=\left[d_{i, j}^{(n-j q)\left(k_{m} L+1\right)+1} d_{i, j}^{(n-j q)\left(k_{m} L+1\right)+2}\right. & \\
& \left.\cdots d_{i, j}^{(n-j q+1)\left(k_{m} L+1\right)}\right], \quad j q \leq n \leq(j+1) q-1, \\
& j T_{k_{f}} \leq t \leq(j+1) T_{k_{f}} . \quad \text { (19) }
\end{aligned}
$$

We can easily find $\boldsymbol{G}^{r}(D)$ and $\boldsymbol{Z}_{L_{t}}^{q}(D)$ using the method in [18]. The overall encoder input during the interval $j T_{k_{f}} \leq t \leq$ $(j+1) T_{k_{f}}$ is $\boldsymbol{a}_{j}$ and its output is $\boldsymbol{d}_{j}$. During the $n$th symbol interval $\left(T=\frac{T_{k_{f}}}{q}\right)$, the $i$ th sampler selects the $L k_{m}+1$-symbol vector $\boldsymbol{X}_{i, n}$, which is input into the $i$ th memoryless modulator.

\section{A. Overall Encoder Complexity}

The number of states in the overall trellis is given by

$$
S_{J}=S_{G} n_{s} \leq S_{G} S_{Z}
$$

where $S_{G}$ and $S_{Z}=\left(p M^{L-1}\right)^{L_{t}}$ are the number of states of the encoders $\boldsymbol{G}^{r}(D)$ and $\boldsymbol{Z}_{L_{t}}^{q}(D)$, respectively. The value of $n_{s}$ is determined by how the states of the encoders $\boldsymbol{G}^{r}(D)$ and $\boldsymbol{Z}_{L_{t}}^{q}(D)$ merge in the overall trellis [18], [23].

We now consider the number of states in the overall encoder for the special case of full response $(L=1) \mathrm{CPM}$, where $n_{s} \leq$ $p^{L_{t}}$. We assume that the space-time encoder is rate- $k / l$ and that $l$ is a multiple of $k_{m} L_{t}$, such that $l=q k_{m} L_{t}$. Then, $n_{s}$ is equal to the number of possible combinations that the $L_{t}$ elements $\left\{c_{j}^{q k_{m}}, c_{j}^{2 q k_{m}}, \ldots, c_{j}^{L_{q} q k_{m}}\right\}$ of the space-time encoder output vector $\left(\boldsymbol{c}_{j}\right)$ can take on before the space-time encoder merges to the zero state.

\section{Simulation Results AND Discussion}

The simulations were run using a frame length of 130 symbols. Each receive antenna receives a faded superposition of the $L_{t}$ simultaneously transmitted signals corrupted by additive white Gaussian noise (AWGN). The fading is assumed to be slow Rayleigh flat fading, such that the $L_{t} L_{r}$ fading gains are constant during a frame, but vary from frame to frame. The random fading gain between each transmit and receive antenna during a frame is modeled as an independent complex Gaussian random variable with zero mean and a variance of $1 / 2$ per dimension. The AWGN noise component is an independent sample of a zero-mean complex Gaussian random process, with power spectral density $N_{0}$. The receiver implements matched filtering and decodes on the overall trellis using the Viterbi algorithm. Perfect channel state information is assumed at the receiver. For simplicity, we restrict the simulations to continuous phase frequency shift keying (CPFSK), a full response CPM $(L=1)$ with a rectangular frequency pulse.

Fig. 3 shows the frame error rate performance of 4-ary CPFSK schemes with two transmit antennas. The space-time codes are $\boldsymbol{G}(D)=\left[1 \frac{1}{1+2 D}\right], \boldsymbol{G}(D)=[D 1]$, and $\boldsymbol{G}(D)=$ $\left[1 \frac{1+2 D}{1+D}\right]$. The rank $(\rho)$ of the signal distance matrix for $\boldsymbol{G}(D)=\left[1 \frac{1}{1+2 D}\right]$ is unity and $d_{\min }^{2}=2.88$. Here, $S_{G}=4$ and $n_{s}=2$, and hence, the STC-CPFSK scheme has an 8state trellis. The code $G(D)=[D 1]$, which is also known as 


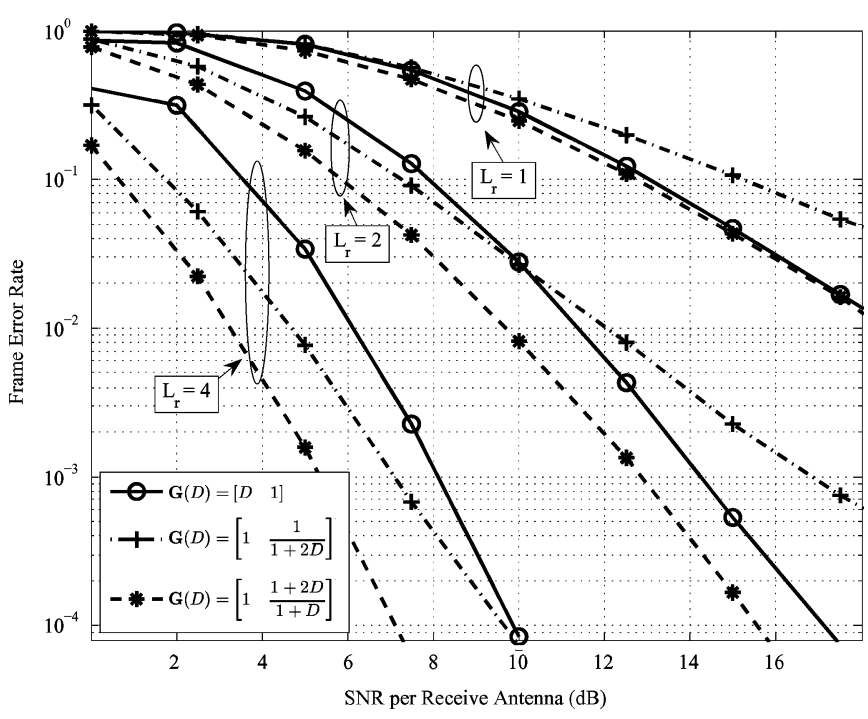

Fig. 3. Frame error rate performance of two transmit antenna systems with 4-CPFSK and one, two, and four receive antennas. The space-time codes are $\boldsymbol{G}(D)=\left[\begin{array}{ll}D & 1\end{array}\right](\circ), \boldsymbol{G}(D)=\left[1 \frac{1}{1+2 D}\right](+)$, and $\boldsymbol{G}(D)=\left[1 \frac{1+2 D}{1+D}\right](*)$.

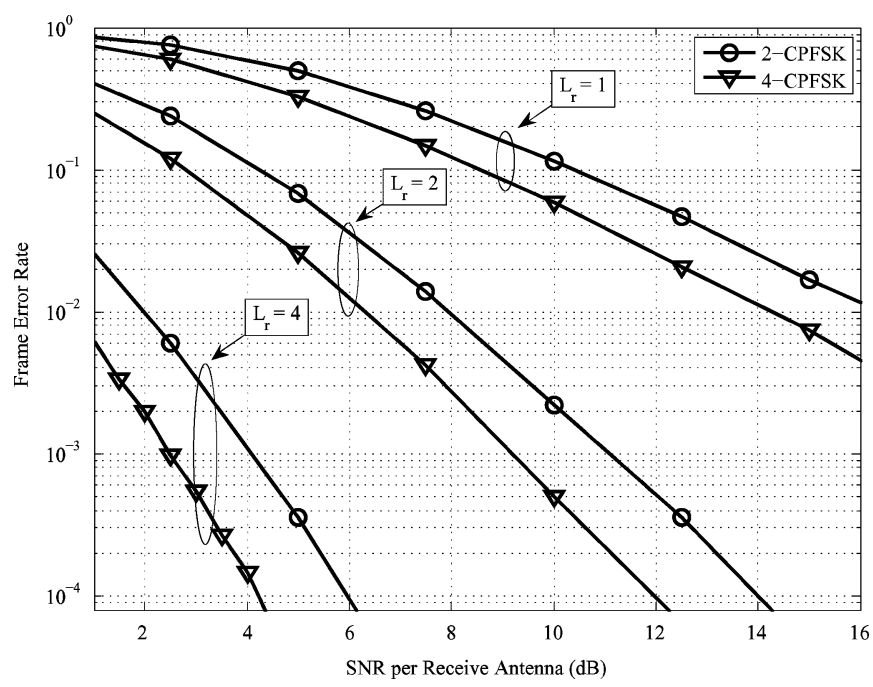

Fig. 4. Frame error rate performance of rate-1 two transmit antenna systems. The systems are 2-CPFSK with $\boldsymbol{G}(D)=[D 1]$ (०) and 4-CPFSK with $\boldsymbol{G}(D)=$ $\left[2+3 D+2 D^{2} 1+D 2+2 D^{2} \frac{3+2 D^{2}}{1+D}\right](\nabla)$ with one, two, and four receive antennas. Both systems yield the same throughput.

delay diversity, is a full rank code $(\rho=2)$ with $d_{\min }^{2}=1.45$ and a 16-state overall trellis $\left(S_{G}=4, n_{s}=4\right)$. The code $\boldsymbol{G}(D)=\left[1 \frac{1+2 D}{1+D}\right]$ is also full rank and has a 16-state trellis $\left(S_{G}=4, n_{s}=4\right)$ with $d_{\min }^{2}=3.24$. The results are presented for one, two, and four receive antennas.

When $\rho L_{r} \geq 4, d_{\min }^{2}$ dominates the error performance. For $\boldsymbol{G}(D)=\left[1 \frac{1}{1+2 D}\right]$, this holds if $L_{r} \geq 4$ and for both the rank-2 codes if $L_{r} \geq 2$. The slopes of the frame error curves of the rank1 STC are less steep than those of the rank-2 codes. However, for $L_{r}=4$, the rank-1 code outperforms the full rank delay diversity system for FER $<10^{-4}$. The two rank- 2 codes have similar performance with one receive antenna. As expected, for
$L_{r} \geq 2$ receive antennas, $\boldsymbol{G}(D)=\left[1 \frac{1+2 D}{1+D}\right]$ with a larger value of $d_{\mathrm{min}}^{2}$ has superior performance.

As a further example, we consider the rate- $\frac{1}{4}$ space-time code

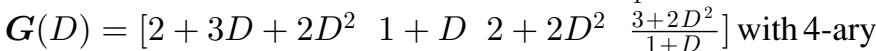
CPFSK and $L_{t}=2$. This system has a throughput of 1 bit per channel use. The space-time encoder has $M^{3}$ states, and with $L_{t}=2$ and CPFSK, the variable $n_{s}=1$. The overall encoder with 4-ary CPFSK then has 64 states. The Euclidean distance of the scheme is 6.94 compared to 2 for delay diversity with 2-ary CPFSK (which has the same rank and throughput) and its frame error rate performance is significantly better as shown in Fig. 4. The cost of the improvement is the number of states in the overall trellis, i.e., 64 compared to 4 . The bit error rate performance (not shown) of the more complex code is worse than for delay diversity when $L_{r}=1$ and better when $L_{r} \geq 2$, this is attributable to the system having $\rho L_{r}<4$ for $L_{r}=1$.

\section{CONCLUSION}

In this paper, we have developed an STC-CPM model that allows the space-time encoder and the continuous phase encoders to be simply combined into a single trellis encoder. This can easily be extended to incorporate external error correction encoding of the same form. The STC-CPM codes can be used as the inner code of an interleaved iteratively decoded system. The model makes it straightforward to calculate $d_{\mathrm{min}}^{2}$, which is important for code design when $\rho L_{r} \geq 4$. Simulation results of selected STC-CPFSK systems are presented. These illustrate the importance of $d_{\mathrm{min}}^{2}$ and the rank of the signal distance matrix.

\section{REFERENCES}

[1] V. Tarokh, N. Seshadri, and A. R. Calderbank, "Space-time codes for high data rate wireless communication: Performance analysis and code construction," IEEE Trans. Inf. Theory, vol. 44, no. 2, pp. 744-765, Mar. 1998.

[2] J.-C. Guey, M. P. Fitz, M. R. Bell, and W.-Y. Kuo, "Signal design for transmitter diversity wireless communication systems over Rayleigh fading channels," in Proc. Veh. Tech. Conf., vol. 46, Atlanta, GA, 1996, pp. 136-140.

[3] X. Zhang and M. P. Fitz, "Constant envelope space-time modems," in Proc. Veh. Tech. Conf., Oct. 2003, vol. 3, pp. 1772-1776.

[4] J. K. Cavers, "Space-time coding using MSK," IEEE Trans. Wireless Commun., vol. 4, no. 1, pp. 185-191, Jan. 2005.

[5] R. L. Maw and D. P. Taylor, "Externally encoded space-time coded systems with continuous phase frequency shift keying," in Proc. Int. Conf. Wireless Netw., Commun. Mobile Comput., Jun. 2005, vol. 2, pp. 15971602.

[6] R. L. Maw and D. P. Taylor, "Space-time coded systems with continuous phase frequency shift keying," in Proc. Global Telecommun. Conf., Nov. 2005, vol. 3, pp. 1581-1586.

[7] J. B. Anderson, T. Aulin, and C.-E. W. Sundberg, Digital Phase Modulation. New York: Plenum, 1986.

[8] X. Zhang and M. P. Fitz, "Space-time code design with CPM transmission," in Proc. Int. Symp. Inf. Theory, Washington, DC, Jun. 2001, p. 327.

[9] X. Zhang and M. P. Fitz, "Space-time code design with continuous phase modulation," IEEE J. Sel. Areas Commun., vol. 21, no. 5, pp. 783-792, Jun. 2003.

[10] J. Sykora, "Constant envelope space-time modulation trellis code design for Rayleigh flat fading channel," in Proc. Global Telecommun. Conf., San Antonio, TX, 2001, vol. 2, pp. 1113-1117.

[11] X. Zhang and M. P. Fitz, "Soft-output demodulator in space-time-coded continuous phase modulation," IEEE Trans. Signal Process., vol. 50, no. 10, pp. 2589-2598, Oct. 2002. 
[12] C.-C. Chen and C.-C. Lu, "Space-time code design for CPFSK modulation over frequency-nonslective fading channels," IEEE Trans. Commun., vol. 53, no. 9, pp. 1477-1489, Sep. 2005.

[13] D. Bokolamulla and T. M. Aulin, "Performance of space-time coded continuous phase modulated signals over different fading environments," in Proc. Int. Symp. Inf. Theory, Sep. 2005, pp. 1048-1052.

[14] W. Zhao and G. B. Giannakis, "Reduced complexity receivers for layered space-time CPM," in IEEE Trans. Wireless Commun., vol. 4, no. 2, pp. 574-582, Mar. 2005.

[15] B. E. Rimoldi, "A decomposition approach to CPM," IEEE Trans. Inf. Theory, vol. 34, no. 2, pp. 260-270, Mar. 1988.

[16] J. L. Massey and T. Mittelholzer, "Convolutional codes over rings," in Proc. 4th Joint Swedish-USSR Workshop Inf. Theory, Gotland, Sweden, Aug.-Sep. 1989, pp. 14-18.

[17] J. L. Massey and T. Mittelholzer, "Systematicity and rotational invariance of convolutional codes over rings," in Proc. 2nd Int. Workshop Algebraic Combinatorial Coding Theory, Leningrad, Russia, Sep.1990, pp. 154 159 .
[18] R. H.-H. Yang and D. P. Taylor, "Trellis-coded continuous-phase frequency-shift keying with ring convolutional codes," IEEE Trans. Inf. Theory, vol. 40, no. 4, pp. 1057-1067, Jul. 1994.

[19] B. E. Rimoldi and Q. Li, "Coded continuous phase modulation using ring convolutional codes," IEEE Trans. Commun., vol. 43, no. 11, pp. 2714 2720, Nov. 1995.

[20] M. Xiao and T. M. Aulin, "Serially concatenated continuous phase modulation with convolutional codes over rings," IEEE Trans. Commun., vol. 54, no. 8, pp. 1387-1395, Aug. 2006.

[21] Z. Chen, J. Yuan, and B. Vucetic, "An improved space-time trellis coded modulation scheme on slow Rayleigh fading channels," in Proc. Int. Conf. Commun., Jun. 2001, vol. 4, pp. 1110-1116.

[22] B. Vucetic and J. Yuan, Space-Time Coding. Chichester: Wiley, 2003.

[23] F. Morales-Moreno, W. Holubowicz, and S. Pasupathy, "Optimization of trellis coded TFM via matched codes," IEEE Trans. Commun., vol. 42, no. 2-4, pp. 1586-1594, Feb./Mar./Apr. 1994. 